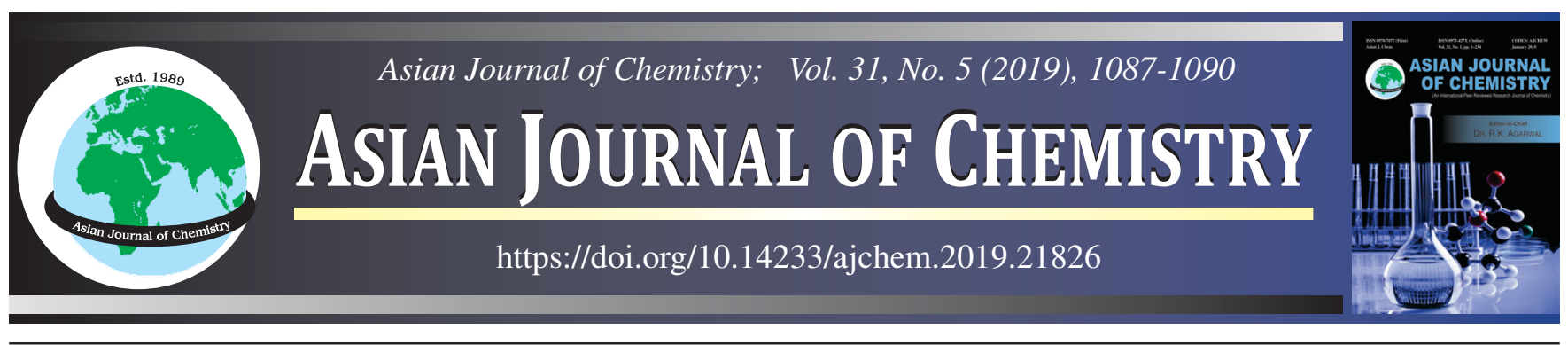

\title{
Microwave Irradiative Synthesis of Triazine Substituted Pyrazoles and Study of Antitubercular and Antimicrobial Activities
}

\author{
Pradip P. Deohate* and Roshani S. Mulani
}

Department of Chemistry, Shri Radhakisan Laxminarayan Toshniwal College of Science, Akola-444001, India

*Corresponding author: E-mail: pradip222091@yahoo.co.in

Received: 20 November 2018;

Accepted: 3 January 2019;

Published online: 28 March 2019;

AJC-19335

Microwave irradiative synthesis of triazine substituted pyrazoles i.e. (4-benzylideneamino-6-methyl-[1,3,5]-triazin-2-yl)-(5-methyl-2-
substituted benzoyl/isonicotinoyl/cinnamoyl-pyrazol-3-yl)-amines have been achieved by the cyclocondensation of N-(4-benzylideneamino-
6-methyl-[1,3,5]-triazin-2-yl)-3-oxo butyramide with substituted acid hydrazides. Synthesis of required butyramide was done by reacting
2,4-diamino-6-methyl-[1,3,5]-triazine with benzaldehyde and then condensing the product with ethyl acetoacetate. Structural investigation
of synthesized compounds has been done by chemical transformation, elemental analysis and IR, ${ }^{1} \mathrm{H}$ NMR, mass spectral studies. Study
of antitubercular and antimicrobial activity of title compounds against some selected Gram-positive and Gram-negative microorganisms
was performed to establish the relationship between structure and activity of compound.

| Keywords: Microwave synthesis, Triazine, Pyrazole, Antitubercular, Antimicrobial study.

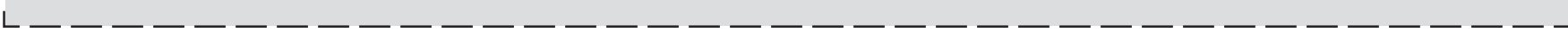

\section{INTRODUCTION}

Microwave irradiation technique has so many advantages over the conventional heating in synthesis of organic compounds [1]. High density microwave irradiation technique can be used for parallel high speed synthesis of number of bioactive compounds [2]. The pharmacophore pyrazole has different practical applications in synthetic organic chemistry [3]. Pyrazole fused heterocyclics have been widely used in pesticides and medicines [4]. Literature has been enriched with the progressive findings about the synthesis and activities of pyrazole which covers the domains like antitubercular [5,6], antitumor [7], antimicrobial [8], antipyretic [9], analgesic [10], ulcerogenic [11], antiinflammatory [12] and anticancer [13]. It was observed that positions $\mathrm{N}-1, \mathrm{C}-3, \mathrm{C}-4$ are much more important for the study of relationship between structure and activity of compound and position C-3 should be linked to different heterocycles for better chemotherapeutic activities [14]. The presence of two bioactive molecules within a single compound increases the antimicrobial activity of that compound. The most common method used for synthesis of pyrazoles is the reaction of 1,3dicarbonyl, oxo-amide, hydrazine hydrate, ester using suitable catalyst [15]. The double nucleophilic character of hydrazine for reaction with each carbonyl group of 1,3-diketone requires long period with high temperature [16].

With these observations, we report herein the synthesis of triazine substituted pyrazoles at C-3 position i.e. (4-benzylideneamino-6-methyl-[1,3,5]-triazin-2-yl)-(5-methyl-2-substituted benzoyl/isonicotinoyl/cinnamoyl-pyrazol-3-yl)-amines (5a-f) by microwave irradiation technique without the use of any catalyst. So as to establish the relationship between structure and activity of compound, all synthesized compounds were studied for their antitubercular and antimicrobial activity against selected microorganisms.

\section{EXPERIMENTAL}

All reactions were performed by the microwave irradiation using commercially available microwave oven. Chemicals used were of A.R. grade. Purity of the compounds was checked on silica gel-G plates by TLC and spots were visualized by iodine vapours. Melting points were recorded using Veego, VMP-D digital melting point apparatus and are uncorrected. ${ }^{1} \mathrm{H}$ NMR spectra were recorded using $\mathrm{CDCl}_{3}$ and DMSO- $d_{6}$ as solvents and TMS as internal standard on Bruker Avance-II 400 NMR spectrometer. IR spectra were recorded in the range 4000-400

This is an open access journal, and articles are distributed under the terms of the Creative Commons Attribution-NonCommercial-ShareAlike 4.0 (CC BY-NC-SA 4.0) International License which allows readers to freely read, download, copy, distribute, print, search, or link to the full texts of its articles and to use them for any other lawful non-commercial purpose as long as the original source is duly acknowledged. 
$\mathrm{cm}^{-1}$ on Perkin-Elmer spectrophotometer. Mass spectral measurements were carried out by EI method at $70 \mathrm{eV}$ on Jeol-JMC 300 spectrometer.

Preparation of 2-amino-4-benzylideneamino-6-methyl[1,3,5]-triazine (2): The compound 2-amino-4-benzylideneamino-6-methyl-[1,3,5]-triazine (2) was prepared by irradiating the mixture of 2,4-diamino-6-methyl-[1,3,5]-triazine (1) $(0.01 \mathrm{~mol})$ and benzaldehyde $(0.01 \mathrm{~mol})$ for $3.5 \mathrm{~min}$ using microwave under solvent free conditions. The crude solid mass obtained was crystallized form hot ethanol, (95\%), m.p. $146^{\circ} \mathrm{C}$ (Found: C, 60.02; H, 5.19; N, 32.81. Calcd. for $\mathrm{C}_{11} \mathrm{H}_{11} \mathrm{~N}_{5}$ : C, 61.97; H, 5.16; N, $32.86 \%) ;{ }^{1} \mathrm{H}$ NMR: $\delta\left(\mathrm{CDCl}_{3}+\mathrm{DMSO}-d_{6}\right)$ $7.94(1 \mathrm{H}, \mathrm{s}, \mathrm{Ar}-\mathrm{CH}=\mathrm{N}), 7.90$ (2H, s, Trz-NH$), 6.90-7.58(5 \mathrm{H}$, $\mathrm{m}, \mathrm{Ar}-\mathrm{H}), 2.27\left(3 \mathrm{H}, \mathrm{s}, \mathrm{Trz}-\mathrm{CH}_{3}\right)$ [17,18]. Preparation of the compound was monitored by TLC using silica gel-G plates and the pure compound was separated using the technique of column chromatography.

Preparation of N-(4-Benzylideneamino-6-methyl[1,3,5]-triazin-2-yl)-3-oxo butyramide (3): The compound $\mathrm{N}$-(4-benzylideneamino-6-methyl-[1,3,5]-triazin-2-yl)-3-oxo butyramide (3) was prepared by treating 2-amino-4-benzylideneamino-6-methyl- [1,3,5]-triazine (2) (0.01 mol) with ethyl acetoacetate $(0.01 \mathrm{~mol})$ for $3 \mathrm{~min}$ by microwave irradiation, the resulting solid was crystallized from hot ethanol, (92\%), m.p. $128{ }^{\circ} \mathrm{C}$ (Found: C, 59.98; H, 5.08; N, 23.52. Calcd. for $\mathrm{C}_{15} \mathrm{H}_{15} \mathrm{~N}_{5} \mathrm{O}_{2}$ : C, 60.60; H, 5.09; N, $23.55 \%$ ); IR (KBr, $v_{\max }$, $\left.\mathrm{cm}^{-1}\right)$ : $3329(\mathrm{NH}), 1681(\mathrm{C}=\mathrm{O}), 1546(\mathrm{C}=\mathrm{N}), 1325(\mathrm{C}-\mathrm{N}) ;{ }^{1} \mathrm{H}$ NMR: $\delta\left(\mathrm{CDCl}_{3}+\mathrm{DMSO}-d_{6}\right) 7.94(1 \mathrm{H}, \mathrm{s}, \mathrm{Ar}-\mathrm{CH}=\mathrm{N}), 7.93$ (1H, s, Trz-NH), 7.38-7.52 (5H, m, Ar-H), 3.46 (2H, s, CO$\mathrm{CH}_{2}-\mathrm{CO}$ ), 2.21 (3H, s, Trz-CH 3 ), 2.19 (3H, s, CO-CH 3 ). Completion of the reaction was monitored with TLC.

Preparation of (2-benzoyl-5-methyl-pyrazol-3-yl)-(4benzylideneamino-6-methyl-[1,3,5]-triazin-2-yl)-amine (5a): The compound (2-benzoyl-5-methyl-pyrazol-3-yl)-(4benzylideneamino-6- methyl-[1,3,5]-triazin-2-yl)-amine (5a) was prepared by microwave irradiative cyclocondensation of mixture of N-(4-benzylideneamino-6-methyl-[1,3,5]-triazin2-yl)- 3-oxo-butyramide (3) (0.01 mol) and benzoic acid hydrazide (4a) $(0.01 \mathrm{~mol})$ for $35 \mathrm{~s}$. The crude solid residue obtained was crystallized from hot ethanol, 5a (92 \%), m.p. $86^{\circ} \mathrm{C}$ (Found: C, 66.12; H, 4.77; N, 24.44. Calcd. for $\mathrm{C}_{22} \mathrm{H}_{19} \mathrm{~N}_{7} \mathrm{O}: \mathrm{C}$, 66.49; H, 4.82; N, $24.67 \%$ ); IR (KBr, $\left.v_{\max }, \mathrm{cm}^{-1}\right)$ : $3298(\mathrm{NH})$, $1683(\mathrm{C}=\mathrm{O}), 1544(\mathrm{C}=\mathrm{N}), 1323(\mathrm{C}-\mathrm{N}), 1176(\mathrm{~N}-\mathrm{N}) ;{ }^{1} \mathrm{H} \mathrm{NMR}$ : $\delta\left(\mathrm{CDCl}_{3}+\mathrm{DMSO}-d_{6}\right) 7.95(1 \mathrm{H}, \mathrm{s}, \mathrm{Ar}-\mathrm{CH}=\mathrm{N}), 7.28-7.94(10 \mathrm{H}$, m, Ar-H), 6.56 (1H, s, Pyrz-H), 4.24 (1H, s, Trz-NH), 2.06 $\left(6 \mathrm{H}, \mathrm{s}, \mathrm{Pyrz}-\mathrm{CH}_{3}, \mathrm{Trz}-\mathrm{CH}_{3}\right) ;{ }^{13} \mathrm{C} \mathrm{NMR}: \delta\left(\mathrm{CDCl}_{3}+\mathrm{DMSO}-d_{6}\right)$ 111.76-140.94 (19C, m, Ar-C, Pyrz-C, Trz-C, CH=N), 156.37 (1C, s, CO), 20.79, 20.56 (2C, s, Pyrz-CH $\left.\mathrm{CH}_{3}, \mathrm{Trz}-\mathrm{CH}_{3}\right)$; MS: $\mathrm{m} / \mathrm{z} 397\left(\mathrm{M}^{+}\right), 382\left(\mathrm{M}^{+}-\mathrm{CH}_{3}\right), 293\left(\mathrm{M}^{+}-\mathrm{C}_{6} \mathrm{H}_{5} . \mathrm{CH}=\mathrm{N}\right), 292\left(\mathrm{M}^{+}-\right.$ $\left.\mathrm{C}_{6} \mathrm{H}_{5} \mathrm{CO}\right), 212\left(\mathrm{M}^{+}-\mathrm{CH}_{3} \mathrm{C}_{6} \mathrm{H}_{5} \mathrm{COC}_{3} \mathrm{HN}_{2}\right), 200\left(\mathrm{CH}_{3} \mathrm{C}_{6} \mathrm{H}_{5} \mathrm{CO}\right.$ $\left.\mathrm{C}_{3} \mathrm{HN}_{2} \mathrm{NH}^{+}\right), 105\left(\mathrm{C}_{6} \mathrm{H}_{5} \cdot \mathrm{CO}^{+}\right), 77\left(\mathrm{C}_{6} \mathrm{H}_{5}^{+}\right)$. This reaction was extended to synthesize other compounds (5b-f) using different substituted acid hydrazides (4b-f): 5b (90 \%), m.p. $72{ }^{\circ} \mathrm{C}$ (Found: C, 66.81; H, 5.11; N, 23.69. Calcd. for $\mathrm{C}_{23} \mathrm{H}_{21} \mathrm{~N}_{7} \mathrm{O}: \mathrm{C}$, 67.14; H, 5.14; N, $23.83 \%$ ); IR (KBr, $\left.v_{\max }, \mathrm{cm}^{-1}\right): 3296(\mathrm{NH})$, $1681(\mathrm{C}=\mathrm{O}), 1541(\mathrm{C}=\mathrm{N}), 1325(\mathrm{C}-\mathrm{N}), 1170(\mathrm{~N}-\mathrm{N}) ;{ }^{1} \mathrm{H}$ NMR: $\delta\left(\mathrm{CDCl}_{3}+\mathrm{DMSO}-d_{6}\right) 7.94(1 \mathrm{H}, \mathrm{s}, \mathrm{Ar}-\mathrm{CH}=\mathrm{N}), 7.07-7.83(9 \mathrm{H}$, m, Ar-H), 6.57 (1H, s, Pyrz-H), 4.45 (1H, s, Trz-NH), 2.34
(3H, s, Ar- $\left.\mathrm{CH}_{3}\right), 2.06$ (6H, s, Pyrz- $\left.\mathrm{CH}_{3}, \mathrm{Trz}_{-} \mathrm{CH}_{3}\right) ;{ }^{13} \mathrm{C} \mathrm{NMR}$ : $\delta\left(\mathrm{CDCl}_{3}+\mathrm{DMSO}-d_{6}\right)$ 111.76-140.29 (19C, m, Ar-C, Pyrz-C, Trz-C, CH=N), 156.91 (1C, s, CO), 28.05 (3C, s, Ar- $\mathrm{CH}_{3}, \mathrm{Pyrz}_{-}$ $\mathrm{CH}_{3}$, Trz- $\left.\mathrm{CH}_{3}\right)$; MS: m/z $410\left(\mathrm{M}^{+}-\mathrm{H}\right), 396\left(\mathrm{M}^{+}-\mathrm{CH}_{3}\right), 320\left(\mathrm{M}^{+}-\right.$ $\left.\mathrm{CH}_{3} \mathrm{C}_{6} \mathrm{H}_{4}\right), 307\left(\mathrm{M}^{+}-\mathrm{C}_{6} \mathrm{H}_{5} \mathrm{CH}=\mathrm{N}\right), 292\left(\mathrm{M}^{+}-\mathrm{CH}_{3} \mathrm{C}_{6} \mathrm{H}_{4} \mathrm{CO}\right), 199$ $\left(\mathrm{CH}_{3} \mathrm{CH}_{3} \mathrm{C}_{6} \mathrm{H}_{4} \mathrm{COC}_{3} \mathrm{HN}_{2}{ }^{+}\right), 119\left(\mathrm{CH}_{3} \mathrm{C}_{6} \mathrm{H}_{4} \mathrm{CO}^{+}\right), 91\left(\mathrm{CH}_{3} \mathrm{C}_{6} \mathrm{H}_{4}^{+}\right)$; c $(95 \%)$, m.p. $94{ }^{\circ} \mathrm{C}$ (Found: C, 63.62; H, 4.50; N, 23.65. Calcd. for $\mathrm{C}_{22} \mathrm{H}_{19} \mathrm{~N}_{7} \mathrm{O}_{2}$ : 63.91; H, 4.63; N, 23.71\%); IR ( $\mathrm{KBr}$, $\left.v_{\max }, \mathrm{cm}^{-1}\right): 3500(\mathrm{OH}), 3379(\mathrm{NH}), 1680(\mathrm{C}=\mathrm{O}), 1548(\mathrm{C}=\mathrm{N})$, $1323(\mathrm{C}-\mathrm{N}), 1168(\mathrm{~N}-\mathrm{N}) ;{ }^{1} \mathrm{H}$ NMR: $\delta\left(\mathrm{CDCl}_{3}+\mathrm{DMSO}-d_{6}\right)$ $7.96(1 \mathrm{H}, \mathrm{s}, \mathrm{Ar}-\mathrm{CH}=\mathrm{N}), 6.98-7.57(9 \mathrm{H}, \mathrm{m}, \mathrm{Ar}-\mathrm{H}), 6.76(1 \mathrm{H}, \mathrm{s}$, Ar-OH), 6.73 (1H, s, Pyrz-H), 4.03 (1H, s, Trz-NH), 2.14 (6H, s, Pyrz- $\mathrm{CH}_{3}$, Trz- $\left.\mathrm{CH}_{3}\right) ;{ }^{13} \mathrm{C} \mathrm{NMR}: \delta\left(\mathrm{CDCl}_{3}+\mathrm{DMSO}-d_{6}\right)$ 121.04-134.07 (19C, m, Ar-C, Pyrz-C, Trz-C, CH=N), 156.41 (1C, s, CO), 13.72 (2C, s, Pyrz- $\mathrm{CH}_{3}$, Trz- $\mathrm{CH}_{3}$ ); d (90\%), m.p. $170{ }^{\circ} \mathrm{C}$ (Found: C, 63.74; H, 4.80; N, 27.07. Calcd. for $\mathrm{C}_{22} \mathrm{H}_{20} \mathrm{~N}_{8} \mathrm{O}$ : C, 64.07; H, 4.89; N, 27.17 \%); IR ( KBr, $\left.v_{\max }, \mathrm{cm}^{-1}\right): 3327$, $3097(\mathrm{NH}), 1680(\mathrm{C}=\mathrm{O}), 1543(\mathrm{C}=\mathrm{N}), 1323(\mathrm{C}-\mathrm{N}), 1170(\mathrm{~N}-$ $\mathrm{N})$; ${ }^{1} \mathrm{H}$ NMR: $\delta\left(\mathrm{CDCl}_{3}+\mathrm{DMSO}-d_{6}\right)$ 7.39-8.89 (10H, m, Ar$\mathrm{H}, \operatorname{Ar}-\mathrm{CH}=\mathrm{N}), 5.88$ (1H, s, Pyrz-H), 3.43 (1H, s, Trz-NH), $2.50\left(2 \mathrm{H}, \mathrm{s}, \mathrm{Ar}-\mathrm{NH}_{2}\right), 2.20\left(6 \mathrm{H}, \mathrm{s}, \mathrm{Pyrz}-\mathrm{CH}_{3}, \mathrm{Trz}-\mathrm{CH}_{3}\right) ;{ }^{13} \mathrm{C}$ NMR: $\delta\left(\mathrm{CDCl}_{3}+\mathrm{DMSO}-d_{6}\right)$ 112.59-128.05 (19C, m, Ar-C, Pyrz-C, Trz-C, CH=N), 156.66 (1C, s, CO), 22.59 (2C, s, Pyrz$\mathrm{CH}_{3}$, Trz-CH ${ }_{3}$ ); e (88 \%), m.p. $118{ }^{\circ} \mathrm{C}$ (Found: C, 63.19; H, 4.52; N, 28.08. Calcd. for $\mathrm{C}_{21} \mathrm{H}_{18} \mathrm{~N}_{8} \mathrm{O}: \mathrm{C}, 63.31 ; \mathrm{H}, 4.55 ; \mathrm{N}$, $28.12 \%)$; f (85 \%), m.p. $104{ }^{\circ} \mathrm{C}$ (Found: C, 67.86; H, 5.01; N, 23.02. Calcd. for $\mathrm{C}_{24} \mathrm{H}_{21} \mathrm{~N}_{7} \mathrm{O}$ : C, 68.07; H, 5.00; N, 23.15\%). The reaction was monitored on silica gel-G plates by TLC.

\section{RESULTS AND DISCUSSION}

The compound 2-amino-4-benzylideneamino-6-methyl$[1,3,5]$-triazine (2) was synthesized by reacting 2,4-diamino6-methyl-[1,3,5]-triazine (1) $(0.01 \mathrm{~mol})$ with benzaldehyde $(0.01 \mathrm{~mol})$ by microwave irradiation for $3.5 \mathrm{~min}$ under solvent free conditions. Completion of the reaction was monitored with TLC and technique of column chromatography was used to separate the pure compound (Scheme-I).

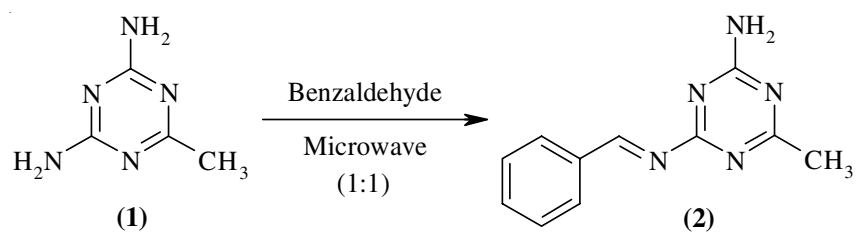

Scheme-I

Compound (2) was then treated with ethyl acetoacetate (0.01 mol) using microwave for $3 \mathrm{~min}$ to give the compound N-(4-benzylideneamino-6-methyl-[1,3,5]- triazin-2-yl)-3-oxo butyramide (3) which on microwave irradiative cyclocondensation with substituted acid hydrazides (4a-f) $(0.01 \mathrm{~mol})$ for 25 to $45 \mathrm{~s}$ afforded (4-benzylideneamino-6-methyl-[1,3,5]triazin-2-yl)-(5-methyl-2-substituted benzoyl/isonicotinoyl/ cinnamoyl-pyrazol-3-yl)-amines (5a-f) (Scheme-II). It was observed that microwave irradiative reactions have high product yield, purity and enhanced reaction rates. IR, ${ }^{1} \mathrm{H}$ NMR and mass spectral investigation of synthesized compounds fully supported the structures and showed single spots in TLC. 


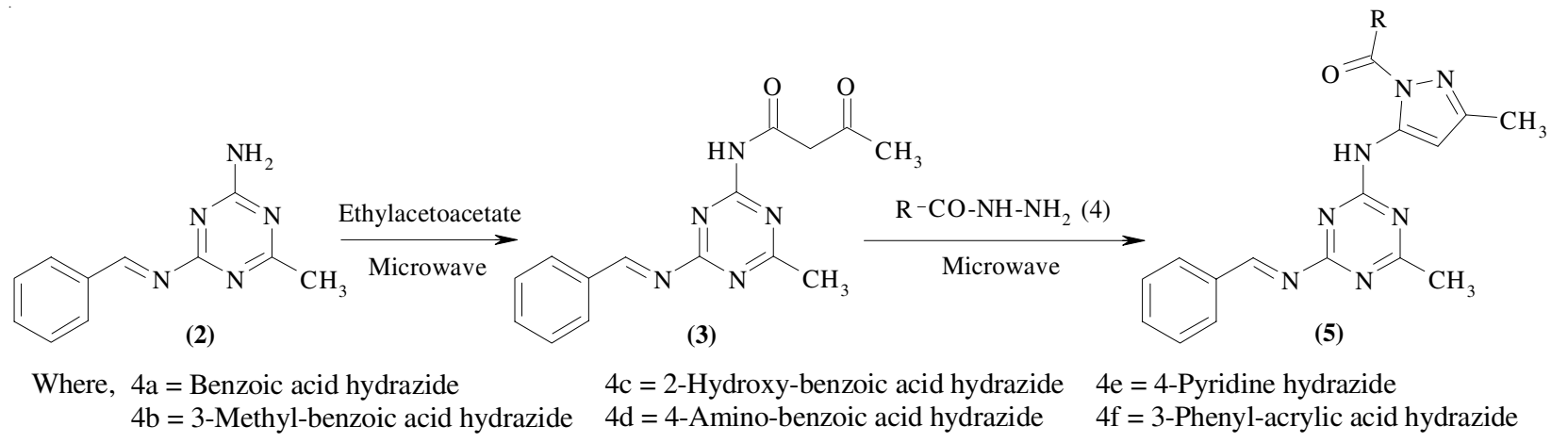

Scheme-II

Antitubercular activity: The compounds (5a-f) have been studied for their in vitro antitubercular activity [19] by microplate alamar blue assay (MABA) method for direct determination of minimum inhibitory concentration (MIC) against $M$. tuberculosis. Test compounds were dissolved in 10 $\%(\mathrm{v} / \mathrm{v})$ DMSO at a concentration of $10 \mathrm{mM}$. Two fold serial dilutions of compounds were made in Middle brook 7H9 medium supplemented with $10 \%(\mathrm{v} / \mathrm{v})$ ADC, in well plates (Nunc) in duplicate. An inoculums of $10^{5} \mathrm{CFU} \mathrm{mL}^{-1}$ was prepared and $200 \mu \mathrm{L}$ was added per well. For each assay, growth controls containing no drug and a sterile control without bacteria were also prepared. The plates were incubated at $37{ }^{\circ} \mathrm{C}$ for 5 days before adding $20 \mu \mathrm{L}$ of sterile $0.01 \%$ resazurin to the wells and then incubated further for $24 \mathrm{~h}$ at $37^{\circ} \mathrm{C}$. The growth of bacteria was indicated by a change in colour from blue to pink i.e. oxidized state to reduced state. The compounds showing MIC at $50 \mu \mathrm{M}$ were further screened for CFU determination using agar dilution method. Serial dilutions of compounds prepared in $0.1 \mathrm{~mL} 10 \%(\mathrm{v} / \mathrm{v})$ DMSO were added to each well of well plates (Nunc). Then $1.9 \mathrm{~mL}$ MB7H10 agar medium supplemented with $10 \%(\mathrm{v} / \mathrm{v})$ OADC were poured to respective wells and allowed to solidify at room temperature. For positive control, streptomycin and rifampin were dissolved in water, filtered, sterilized and used in 6 and $2 \mu \mathrm{g} \mathrm{mL}^{-1}$ respectively. Solution $10 \mu \mathrm{L}$ was inoculated in each well on solidified agar medium and incubated for 4 weeks at $37{ }^{\circ} \mathrm{C}$ to record the growth. The compounds $(\mathbf{5 c}),(\mathbf{5 d})$ and $(\mathbf{5 e})$ showed promising activity against $M$. tuberculosis. MIC values of compounds (5d) and (5e) have been found to be $6.25 \mu \mathrm{M}$ and of compound (5c) was found to be $12.5 \mu \mathrm{M}$ (Table-1).

\begin{tabular}{cccccc}
\multicolumn{7}{c}{ TABLE-1 } \\
ANTITUBERCULAR ACTIVITY OF COMPOUNDS (5a-f) \\
\hline \multirow{2}{*}{ Compd. } & \multicolumn{5}{c}{ Concentration $(\mu \mathrm{M})$} \\
\cline { 2 - 6 } & 3.125 & 6.25 & 12.5 & 25 & 50 \\
\hline $\mathbf{5 a}$ & IA & IA & IA & IA & IA \\
$\mathbf{5 b}$ & IA & IA & IA & IA & IA \\
$\mathbf{5 c}$ & IA & IA & A & A & A \\
$\mathbf{5 d}$ & IA & A & A & A & A \\
$\mathbf{5 e}$ & IA & A & A & A & A \\
$\mathbf{5 f}$ & IA & IA & IA & IA & IA \\
\hline A $=$ Active, IA $=$ Inactive & & &
\end{tabular}

Antimicrobial activity: The compounds (5a-f) have been studied for their antibacterial activity by using cup plate diffusion method $[20,21]$. The bacterial organisms having both
Gram-positive and Gram-negative strains i.e. S. aureus, E. coli, S. typhi, P. vulgaris and B. subtilis were used. Sensitivity plates were seeded with a bacterial innoculum of $1 \times 10^{6} \mathrm{CIU} \mathrm{mL}^{-1}$ and each well of diameter $10 \mathrm{~mm}$ was loaded with $0.1 \mathrm{~mL}$ of test compound solution $\left(1000 \mu \mathrm{g} \mathrm{mL}^{-1}\right)$ in DMF, so that concentration of each test compound was $100 \mu \mathrm{g} \mathrm{mL}^{-1}$. After incubation for $24 \mathrm{~h}$ at $37^{\circ} \mathrm{C}$, the zones of inhibition were recorded using vernier caliper. It was found that the compounds (5d) and (5e) were highly active against $S$. aureus and E. coli and moderately active against $S$. typhi and $P$. vulgaris. Compounds (5c) was moderately active against $S$. aureus and E. coli. Majority of the compounds were found to be inactive against B. subtilis (Table-2). Serial dilution technique [22] using nutrient broth medium was used to determine the MIC values. For compounds (5d) and (5e) MIC values were found to be 50 and $65 \mu \mathrm{g} \mathrm{mL}{ }^{-1}$ respectively against $S$. aureus and 60 and $45 \mu \mathrm{g}$ $\mathrm{mL}^{-1}$ respectively against $E$. coli.

\begin{tabular}{cccccc}
\multicolumn{6}{c}{ TABLE-2 } \\
ANTIMICROBIAL ACTIVITY OF COMPOUNDS (5a-f) \\
\hline Compd. & \multicolumn{5}{c}{ Microorganisms } \\
\cline { 2 - 6 } & S. aureus & E. coli & S. typhi & P. vulgaris & B. subtilis \\
\hline $\mathbf{5 a}$ & + & + & - & - & - \\
$\mathbf{5 b}$ & + & + & + & + & - \\
$\mathbf{5 c}$ & ++ & ++ & + & + & + \\
$\mathbf{5 d}$ & +++ & +++ & ++ & ++ & + \\
$\mathbf{5 e}$ & +++ & +++ & ++ & ++ & + \\
$\mathbf{5 f}$ & + & + & + & + & - \\
\hline+++ & + Highly active $(21 \mathrm{~mm}$ and above); ++ = Moderately active $(16-$ \\
$20 \mathrm{~mm}) ;++=$ Weakly active $(11-15 \mathrm{~mm}) ;-=$ Inactive (10 mm and less)
\end{tabular}

\section{Conclusion}

In present communication synthesis of triazine substituted pyrazoles (5a-f) have been reported by microwave irradiative cyclocondensation. This method was found to the simple, efficient and completed within a very short period of time with good yield. Study of antitubercular and antimicrobial activity of synthesized compounds showed that, compounds (5c), (5d) and (5e) have promising activity against $M$. tuberculosis and compounds (5d) and (5e) were highly active against $S$. aureus and E. coli.

\section{ACKNOWLEDGEMENTS}

The authors are thankful to Director, S.A.I.F, Punjab University, Chandigarh and C.S.I.R. Central Drug Research 
Institute, Lucknow, India for providing analytical, spectral data and antitubercular activity analysis.

\section{CONFLICT OF INTEREST}

The authors declare that there is no conflict of interests regarding the publication of this article.

\section{REFERENCES}

1. P. Lidström, J. Tierney, B. Wathey and J. Westman, Tetrahedron, 57, 9225 (2001);

https://doi.org/10.1016/S0040-4020(01)00906-1.

2. S.L. Cui, X.F. Lin and Y.G. Wang, J. Org. Chem., 70, 2866 (2005); https://doi.org/10.1021/jo047823h.

3. A.A. Elagamy, F.M.A. E1-Taweed, F.A. Amer and H.H. Zoorobs, Arch. Pharma., 246, 320 (1987).

4. A.M.G. Silva, A.C. Tome, M.G. Neves, M.S. Tome and J.A.S. Cavaleiro, Synlett, 1155 (2002); https://doi.org/10.1055/s-2002-32581.

5. D. Castagnolo, A. De Logu, M. Radi, B. Bechi, F. Manetti, M. Magnani, S. Supino, R. Meleddu, L. Chisu and M. Botta, Bioorg. Med. Chem., 16, 8587 (2008); https://doi.org/10.1016/j.bmc.2008.08.016.

6. P.R. Kawle, P.P. Deohate, B.N. Berad, K.K. Srivastava and P. Sharma, Indian J. Chem., 55B, 1531 (2013).

7. H.J. Park, K. Lee, S. Park, B. Ahn, J.C. Lee, H.Y. Cho and K.I. Lee, Bioorg. Med. Chem. Lett., 15, 3307 (2005); https://doi.org/10.1016/j.bmcl.2005.03.082.

8. P.R. Kawle, P.P. Deohate and B.N. Berad, Indian J. Heterocycl. Chem., 23, 253 (2014)

9. F.R. Souza, V.T. Souza, V. Ratzlaff, L.P. Borges, M.R. Oliveira, H.G. Bonacorso, N. Zanatta, M.A.P. Martins and C.F. Mello, Eur. J. Pharmacol., 451, 141 (2002); https://doi.org/10.1016/S0014-2999(02)02225-2.
10. M.M.F. Ismail, Y.A. Ammar, H.S.A. El-Zahaby, S.I. Eisa and S. ElSayed Barakat, Arch. Pharma. Life Sci., 340, 476 (2007); https://doi.org/10.1002/ardp.200600197.

11. A. Mohammad and S. Kumar, Indian J. Chem., 44B, 2532 (2005).

12. S.A.M. El-Hawash, E.-S.A.M. Badawey and I.M. El-Ashmawey, Eur. J. Med. Chem., 41, 155 (2006); https://doi.org/10.1016/j.ejmech.2005.09.006.

13. K.-R. Kim, J.-L. Kwon, J.-S. Kim, Z. No, H.R. Kim and H.G. Cheon, Eur. J. Pharmacol., 528, 37 (2005); https://doi.org/10.1016/j.ejphar.2005.10.027.

14. A. Kimata, H. Nakagawa, R. Ohyama, T. Fukuuchi, S. Ohta, T. Suzuki and N. Miyata, J. Med. Chem., 50, 5053 (2007); https://doi.org/10.1021/jm070688r.

15. L. Knorr, Chem. Ber., 17, 546 (1884); https://doi.org/10.1002/cber.188401701152.

16. K. Makino, H.S. Kim and Y. Kurasawa, J. Heterocycl. Chem., 36, 321 (1999); https://doi.org/10.1002/jhet.5570360202.

17. N.B. Colthup, L.H. Dally and S.E. Wiberly, Introduction to Infrared and Raman Spectroscopy, Academic Press: New York (1964).

18. R.M. Silverstein, G.C. Bassler and T.C. Morrill, Spectrometric Identification of Organic Compounds, John Wiley \& Sons: New York, edn 4 (1981).

19. I.B. Babalola, E.A. Adelakun, Y. Wang and F.O. Shode, J. Pharm. Phytochem., 1, 19 (2012).

20. A.L. Barry, The Antimicrobial Suspectibility Test: Principle and Practices, Illus Lea and Fibiger: Philadephia, PA, U.S.A., p. 180 (1976).

21. F. Cavanagh, Analytical Microbiology, Academic Press: New York, p. 126 (1963).

22. C. Nishina, N. Enoki, S. Tawata, A. Mori, K. Kobayashi and M. Fukushima, Agric. Biol. Chem., 51, 139 (1987); https://doi.org/10.1080/00021369.1987.10867965. 